\title{
Educação entre pares e identificação de riscos durante a adolescênciał uma revisão bibliogräfica
}

\author{
Peer education and risk identification during \\ adolescence: a bibliographical review
}

Nathália Soares De Oliveira ${ }^{1}$, Audrey Vidal Pereira ${ }^{2}$

\begin{abstract}
1. Especialista em Saúde da Família pela Escola de Enfermagem Aurora de Afonso Costa. Universidade Federal Fluminense. Niterói - Rio de Janeiro - Brasil. ORCID: https://orcid.org/0000-0001-8766-9310. Email: nathalia.olisoa@gmail.com

2. Doutor em Saúde Pública. Docente na Escola de Enfermagem Aurora de Afonso Costa. Universidade Federal Fluminense. Niterói - Rio de Janeiro - Brasil. ORCID: http://orcid.org/0000-0002-6570-9016. Email: auviprof@yahoo.com.br
\end{abstract}

CONTATO: Nathália Soares de Oliveira | Rua Castorina Faria Lima, 327 - Ap. 201 - Portuguesa | Ilha do Governador | Rio de Janeiro - RJ | Telefone: (21) 99338-7898 | E-mail: nathalia.olisoa@ gmail.com

COMO CITAR: Oliveira NS, Pereira AV. Educação entre pares e identificação de riscos durante a adolescência: uma revisão bibliográfica. R. Saúde Públ. Paraná. 2020 Jul;3(1):129-136.

RESUMO A Educação entre Pares (EP) tem se mostrado uma estratégia eficaz na identificação de fatores de risco na adolescência. Este trabalho tem como objetivos pesquisar na literatura científica a relação da EP com a identificação de fatores de risco no período da adolescência, bem como elucidar possíveis vantagens e limitações dessa estratégia na atenção básica. O estudo justifica-se por abordar questões que buscam melhorar o processo de transmissão e compreensão de informações, e consequentemente reduzir os fatores de risco desse grupo populacional. A metodologia adotada para o desenvolvimento dos objetivos referidos foi a revisão de literatura bibliográfica, que contou com quatro etapas de seleção até a amostra final de seis artigos. A revisão bibliográfica aponta que a EP, 
quando implementada de maneira adequada, pode ser considerada uma estratégia positiva na identificação e análise dos fatores de riscos durante a adolescência e, de modo consequente, facilitar o processo de ganhos em saúde.

PALAVRAS-CHAVE: Adolescente. Fatores de Risco. Educação entre pares.

\begin{abstract}
Peer education (PE) has been proving to be an effective strategy for the identification of risk factors during adolescence. The objectives of this work are to search, in scientific literature, the relation of PE with the identification of risk factors during adolescence as well as to elucidate possible advantages and limitations of this strategy in basic care. The study is relevant as it approaches issues that aim to improve the processes of information transmission and comprehension, consequently reducing risk factors in the targeted group. The methodology chosen to achieve the referred objectives is the literature review, which followed four stages up to the final sample of six articles being selected. The bibliographical review shows that PE, when properly implemented, can be considered as a positive strategy in the identification and analysis of risk factors during adolescence, and consequently to ease health gain processes.
\end{abstract}

KEYWORDS: Adolescent. Risk Factors. Peer education.

\title{
INTRODUÇÃO
}

A

Educação entre Pares (EP) pode ser entendida como um processo que ocorre durante um período de tempo, através do qual indivíduos capacitados (que não necessariamente se especializaram no tópico, mas tiveram experiências semelhantes) desenvolvem atividades educacionais com o objetivo de exercitar competências de seus pares (iguais) ${ }^{1-2}$. Alguns autores sugerem que as diferenças culturais e de linguagem entre o educador e o usuário do Sistema Único de Saúde (SUS) podem dificultar a transmissão do conhecimento e que a utilização de integrantes da comunidade do próprio indivíduo pode resultar em desfechos de saúde mais favoráveis e com menor custo financeiro ${ }^{2-3}$.

Alguns trabalhos podem ser encontrados com a relação entre a educação entre pares e a identificação de fatores de risco na adolescência. Saúde sexual e reprodutiva, exposição a ISTs/HIV/AIDS, violência, consumo de tabaco, álcool e drogas são as principais temáticas abordadas nesses textos ${ }^{1.4-6}$.

O ambiente escolar tem se mostrado um importante espaço de troca de saberes, visto que a escola pode ser considerada um local privilegiado, uma vez que é a instituição que alcança, de maneira regular, o maior número de adolescentes e jovens ${ }^{6-7}$. 
Para regulamentar as atividades para a saúde no âmbito escolar, o Decreto nº 6.286 de 05 de dezembro de 2007 instituiu o Programa Saúde na Escola (PSE), através de uma política intersetorial do Ministério da Saúde e do Ministério da Educação, que tem como premissa ações que visam implementação, monitoramento e avaliação do projeto, atuando na redução de fatores de risco nesse grupo populacional ${ }^{8}$

Desta forma, este trabalho tem como objetivos pesquisar na literatura científica a relação da educação entre pares com a identificação de fatores de risco no período da adolescência. Assim como, elucidar possíveis vantagens e limitações dessa estratégia na atenção básica. Em uma perspectiva ampliada, o estudo pretende abrir o debate e contribuir para ações socioeducativas voltadas para a redução de riscos nesse grupo. E também apresentar considerações que possam contribuir para a formação da consciência crítica. promovendo aos adolescentes o conhecimento e a valorização do seu protagonismo frente à sua saúde e de sua coletividade.

Neste contexto, buscou-se desenvolver um material teórico que, através de uma revisão bibliográfica, pudesse mostrar o que a literatura dos últimos 10 anos evidencia sobre a relação da educação entre pares e a redução de riscos na adolescência.

\section{MÉTODO}

A metodologia utilizada para o desenvolvimento dos objetivos referidos foi a revisão bibliográfica, que contou com 4 etapas: identificação do problema, formulação do problema, busca de evidências e avaliação das evidências disponíveis na literatura9 . A estratégia PICO foi utilizada para a identificação e formulação do problema, orientando a construção da pergunta da pesquisa e da busca bibliográfica.

A busca bibliográfica eletrônica utilizou as principais bases de dados de saúde pública como: Sistema Online de Busca e Análise de Literatura Médica (MEDLINE), Literatura Latino-Americana e do Caribe em Ciências da Saúde (LILACS) e Scientific Electronic Library Online (SciELO). O período compreendido pela busca foi de 2008 a 2018. Os descritores utilizados foram: adolescência, fatores de risco e educação entre pares (peereducation).

Constituíram a amostragem inicial o quantitativo de 344 artigos: 229 (MEDLINE), 67 (LILACS) e 48 (SciELO). As respectivas bases de dados, descritores associados e quantidade de artigos resultantes após a aplicação dos critérios de inclusão estão representados no Quadro 1.

Foram encontrados 344 artigos. 25 artigos duplicados foram excluídos. Após a etapa, foi realizada uma leitura dos estudos primários por título e resumo, de acordo com os critérios de exclusão e com a questão norteadora do projeto, resultando na exclusão de 228 artigos. Ficaram 91 artigos para análise do texto na integra. Destes, 6 artigos foram selecionados para compor a amostra final, por atender ao objeto norteador proposto pelo estudo.

O fluxograma (Figura 1) abaixo representa o caminho seguido para busca do material a ser discutido nesta revisão. 
Quadro 1: Base de dados, descritores associados e número de artigos encontrados.

\begin{tabular}{|lccc|}
\hline \multicolumn{1}{|c}{ DESCRITORES } & MEDLINE & LILACS & SciELO \\
\hline Adolescência AND Fatores de risco & 8 & 30 & 20 \\
Adolescência AND Educação entre pares & 0 & 1 & 2 \\
Fatores de risco AND Educação entre pares & 0 & 0 & 0 \\
$\begin{array}{l}\text { Adolescência AND Fatores de risco AND Educação } \\
\text { entre pares }\end{array}$ & 0 & 0 & 0 \\
Adolescence AND Risk factors & 219 & 36 & 23 \\
Adolescence AND Peer education & 0 & 0 & 1 \\
Risk factors AND Peer education & 2 & 0 & 0 \\
Adolescence AND Risk factors AND Peer education & 0 & 0 & 2 \\
\hline TotAL & $\mathbf{2 2 9}$ & $\mathbf{6 7}$ & $\mathbf{4 8}$ \\
\hline
\end{tabular}

Fonte: elaborado pelos autores (2019)

Figura 1: Fluxograma do processo de seleção da amostragem final do estudo.

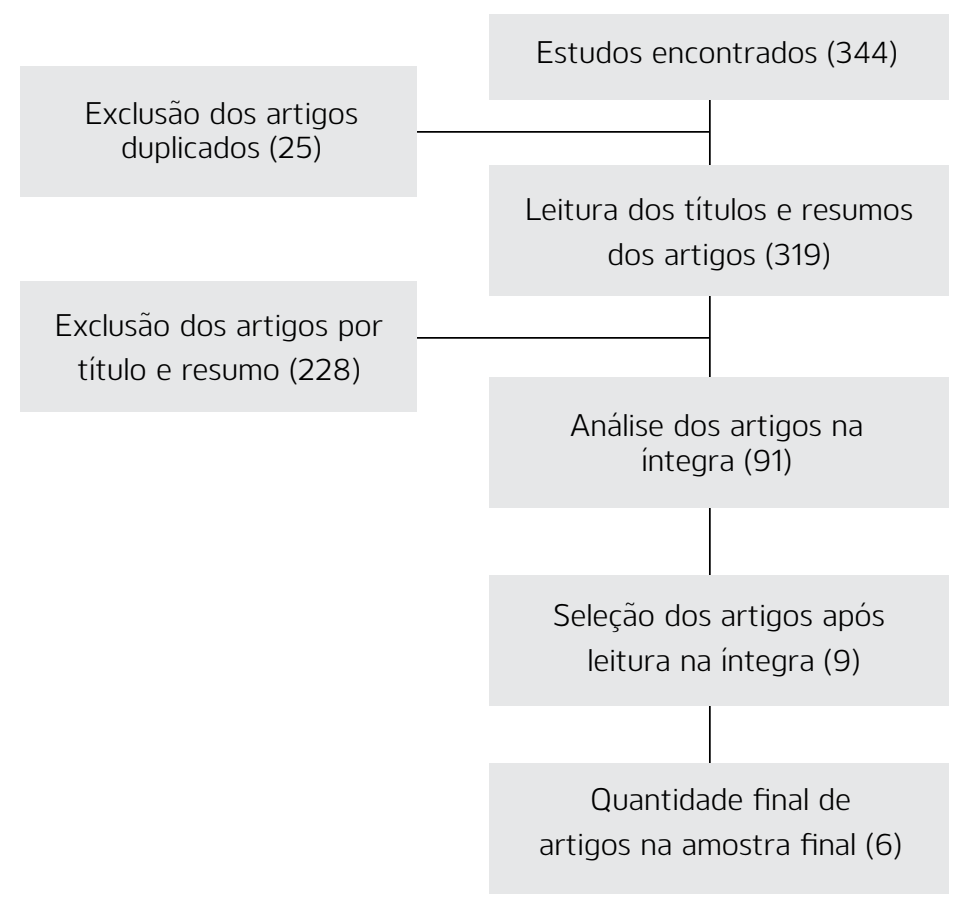

Fonte: elaborado pelos autores (2019) 


\section{RESULTADOS}

A caracterização dos seis artigos selecionados será apresentada no Quadro 2, que expõe dados como autor, revista, cidade, ano, título, objetivos e métodos.

Os fatores de risco citados como interferentes ao desenvolvimento saudável de adolescentes, encontrados nos artigos selecionados e discutidos nesta revisão bibliográfica, foram: gravidez na adolescência, exposição a IST/HIV/AIDS, uso de drogas, suicídio, violência, dietas rigorosas, excesso de exercícios físicos, rejeição familiar, relações de gênero, diversidade sexual, raça e etnia.

Quadro 2: Caracterização dos estudos selecionados no período de 2003 a 2018

\begin{tabular}{|c|c|}
\hline Autor/Local/Periódico/Ano & Título/ Objetivos/ Método \\
\hline $\begin{array}{l}\text { Laperrière H/ Ottawa/ Ciência \& } \\
\text { Saúde Coletiva/ } 2008 .\end{array}$ & $\begin{array}{l}\text { Evaluation of STD/HIV/AIDS peer-education and danger: } \\
\text { a local perspective/ Buscar a compreensão coletiva da } \\
\text { educação por pares em áreas de prostituição, identificar } \\
\text { dimensões coletivas significativas como a história coletiva e } \\
\text { as condições de trabalho perigosas/ Observação participante. }\end{array}$ \\
\hline $\begin{array}{l}\text { Assis SG; Gomes R; Pires TO /Rio } \\
\text { de Janeiro/ Revista Saúde Pública/ } \\
2014 .\end{array}$ & $\begin{array}{l}\text { Adolescência, comportamento sexual e fatores de risco à } \\
\text { saúde/ Analisar a relação entre comportamento sexual e } \\
\text { fatores de risco à saúde física ou mental entre adolescentes/ } \\
\text { Qualiquantitativo. }\end{array}$ \\
\hline $\begin{array}{l}\text { Dias A; Saiter, M; Cunha N/ São } \\
\text { Paulo/ Educare, Revista Científica de } \\
\text { Educação/ } 2015 \text {. }\end{array}$ & $\begin{array}{l}\text { Avaliação de fatores de risco na adolescência/ Identificar } \\
\text { fatores de risco; avaliar eventos e comportamentos de risco } \\
\text { de estudantes-adolescentes paulistas/ Qualiquantitativo. }\end{array}$ \\
\hline $\begin{array}{l}\text { Santos KB; Murta SG/ Brasília/ } \\
\text { Psicologia: Ciência e Profissão/ } 2016 .\end{array}$ & $\begin{array}{l}\text { Influência dos Pares e Educação por Pares na Prevenção à } \\
\text { Violência no Namoro/ Sumarizar aspectos teóricos relativos } \\
\text { à influência dos pares no contexto da violência no namoro, } \\
\text { bem como descrever estudos com foco na prevenção à } \\
\text { violência no namoro, os quais utilizaram, no todo ou em parte, } \\
\text { a estratégia da educação por pares como componente no } \\
\text { desenho dessas intervenções/ Revisão narrativa. }\end{array}$ \\
\hline $\begin{array}{l}\text { Santos MP et al./ Bahia/ Revista } \\
\text { Baiana Enfermagem/ 2017. }\end{array}$ & $\begin{array}{l}\text { Promoção da saúde sexual e reprodutiva de adolescentes: } \\
\text { educação por pares/ Descrever o processo de educação por } \\
\text { pares, desenvolvido por jovens católicos como promotores } \\
\text { da saúde sexual e reprodutiva na adolescência/ Qualitativo. }\end{array}$ \\
\hline $\begin{array}{l}\text { Rosário AC; Candeias A; Melo M/ } \\
\text { Évora/ Revista Psicologia/ } 2017 .\end{array}$ & $\begin{array}{l}\text { Violência entre pares na adolescência: um estudo com } \\
\text { estudantes no início e no final do } 3 .^{\circ} \text { ciclo do ensino básico/ } \\
\text { Caracterizar comportamentos de violência entre pares, } \\
\text { em função do nível de escolaridade, gênero e variáveis } \\
\text { socioafetivas/ Qualiquantitativo. }\end{array}$ \\
\hline
\end{tabular}

Fonte: elaborado pelos autores (2019) 


\section{DISCUSSÃO}

O processo de EP, envolvendo adolescentes e jovens, pode acontecer em qualquer espaço público que possibilite a realização das atividades. Entre esses espaços, a escola é considerada um local privilegiado e estratégico uma vez que é a instituição que alcança, de maneira regular, o maior número de indivíduos desse grupo populacional ${ }^{6,8}$.

Acreditando que adolescente aprende mais com adolescente, o Ministério da Saúde e o Ministério da Educação elaboraram um fascículo intitulado: Metodologia de Educação entre Pares, para subsidiar os educadores entre pares participantes das atividades do PSE. Teatro, dança, música e elementos da cultura popular são usados como formas de abordar, com mais profundidade, os temas de interesse comum.

Foi em temas como IST/HIV/AIDS que a EP ganhou maior destaque1. Laperrière também abordou o assunto, quando em seu artigo Evaluation of STD/HIV/AIDS peer-education and danger: a local perspective, deu destaque a uma avaliação dos projetos de educação entre pares, com trabalhadores do sexo, que possuem uma parcela expressiva de adolescentes marginalizados atuantes. $\mathrm{O}$ que se pode concluir no estudo é que deve se levar em consideração a importância do papel desempenhado pelos profissionais de saúde, no apoio aos educadores de pares, em seus imprevisíveis contextos ${ }^{4}$.

Neste caso, os profissionais de saúde podem incentivar formas de expressão desses educadores em situações marginalizadas; tentando entender a dinâmica, as condições de trabalho local e implicação na educação de pares; propondo soluções construtivas; assim como, incentivar o surgimento de "novas formas de conhecimento" na prevenção de IST/HIV/AIDS4.

Segundo Assis, Gomes e Pires, jovens pertencentes a minorias sexuais - homossexuais ou bissexuais vivenciam mais fatores de risco do que os jovens heterossexuais. Os problemas de saúde encontrados nos estudos com esses jovens são: altos níveis de tentativas de suicídios, uso elevado de substâncias, sintomas de depressão e outros problemas de saúde mental, frequentes comportamentos sexuais de risco, incluindo IST/HIV/AIDS, gravidez na adolescência, abuso físico ou sexual, distúrbios alimentares e rejeição familiar¹0.

Os fatores de riscos à saúde a estes adolescentes podem ser compreendidos a partir do imaginário social, que frequentemente desqualifica suas relações afetivossexuais. Concomitantemente, alguns estudos revelam que adolescentes gays e bissexuais podem não só consumir mais álcool, como também idealizar o suicídio de 2 a 7 vezes mais do que heterossexuais. Assim como, se expor mais a riscos não tanto pela não aceitação de sua orientação sexual, mas pela difícil aceitação por parte de seus familiares e colegas ${ }^{110}$.

Um dos eixos norteadores do PSE é a prevenção dos riscos e danos do uso de drogas, sendo o álcool e o tabaco as drogas que mais contribuem para a mortalidade da população e para os anos de vida perdidos por incapacidade. Alguns estudos ressaltam que o PSE tem mostrado eficácia significativa na redução do consumo e no retardamento do início do consumo de drogas em adolescentes e jovens em fase escolar

Uma pesquisa realizada no Distrito Federal buscou analisar as situações de risco psicossocial de adolescentes ( $n=852$ ). Os resultados evidenciaram que em relação à violência, $22,3 \%$ dos adolescentes tinham alguém da família que já esteve preso, indicando que os processos criminais faziam parte do cotidiano destes adolescentes ${ }^{11}$.

No Brasil, o alvo preferencial de morte por homicídio compreende adolescentes e jovens adultos negros do sexo masculino, em especial procedentes das chamadas classes populares urbanas ${ }^{1.12}$. Nesse contexto, de cada 7 indivíduos assassinados, 5 são negros evidenciando que a cor da pele influencia diretamente a probabilidade de um individuo sofrer homicídio $(78,9 \%)^{11,12}$. 
A literatura também aponta que a influência dos pares desempenha um papel importante na compreensão da violência no namoro e doenças como bulimia e anorexia. E que programas multicomponentes de prevenção à violência no namoro e de doenças causadas por dietas rigorosas, focados no ensino de habilidades, é mais desejável em detrimento de intervenções estritamente informativas. 0 uso de educadores de pares para encorajar comportamentos saudáveis entre adolescentes tem sido um componente comum em programas preventivos nesses casos $^{1.5}$.

Esta revisão de literatura sugere que as iniciativas de EP podem gerar resultados positivos na redução de riscos durante a adolescência, quando realizada de maneira adequada.

Algumas das vantagens dessa estratégia podem ser consideradas o baixo custo financeiro e o processo de transmissão e compreensão de informações, que pode ser facilitado, visto que o educador de pares e os grupos-alvo compartilham de características, interesses e estilos de vida similares. Essas afinidades podem ser consideradas fundamentais no desenvolvimento de estratégias de motivação para as mudanças comportamentais mais efetivas ${ }^{4-6,13}$

Contudo, algumas limitações devem ser ressaltadas. Embora, muitas vezes, alguns autores salientem que a EP seja considerada uma estratégia simples de desenvolver e que exige baixos recursos, é na realidade uma opção complexa. Reforçam, ainda, a importância da elaboração de materiais específicos que possam servir de norteadores para a execução adequada ${ }^{4,5,13}$

Nesse contexto, destaca-se a importância do protagonismo de adolescentes e jovens no processo de construção de novos conhecimentos. A EP pode facilitar a identificação dos fatores de risco e. consequentemente, favorecer o processo de ganhos em saúde ${ }^{4-6.13}$.

Adolescentes que estão inseridos em comunidades que se caracterizam pela percepção positiva, confiança, envolvimento, ajuda mútua, tendem a adotar comportamentos mais positivos de saúde ${ }^{1,4-6,10,13}$.

\section{CONSIDERAÇÕES FINAIS}

A revisão de literatura aponta no sentido de que a Educação entre Pares, quando implementada de maneira adequada, com materiais específicos, que sirvam de norteadores, pode ser considerada uma estratégia positiva na identificação e análise dos fatores de riscos durante a adolescência, e de modo consequente facilitar o processo de ganhos em saúde nesse grupo populacional. Para tanto, é necessário reconhecer a complexidade da estratégia de EP e se beneficiar de suas vantagens, como baixo custo financeiro e maior afinidade entre os adolescentes que ensinam e aprendem a partir das trocas de experiências, a fim de contribuir para comportamentos de saúde mais positivos.

Os fatores de risco citados como interferentes ao desenvolvimento saudável de adolescentes, encontrados nos artigos selecionados e discutidos nesta revisão, como gravidez na adolescência, exposição a ISTs/HIV/AIDS, uso de álcool e drogas, suicídio e violência, podem se beneficiar da EP para a redução desses riscos, e consequentemente, gerar resultados positivos em saúde.

Neste sentido, os principais indivíduos envolvidos podem contribuir para melhorar o acesso e o conhecimento acerca dos diferentes serviços de saúde prestados aos adolescentes e, principalmente. funcionar como um elo de ligação entre os seus pares e o sistema de saúde. No entanto, faz-se necessário continuar a desenvolver pesquisas que permitam identificar através da EP diferentes problemáticas de saúde no período da adolescência. 


\section{REFERÊNCIAS}

1. Dias A, Saiter M, Cunha N. Avaliação de fatores de risco na adolescência. Rev Cient Educ. 2015:1(1):115-32.

2. Molazem Z et al. Effects of a peer-led group education on fear, anxiety and depression levels of patients undergoing coronary angiography. Invest Educ Enferm. 2018;36(1)

3. Grillo MFF et al. Efeito de diferentes modalidades de educação. Rev Ass Med Bras. 2013:59(4):400-5.

4. Laperrière H. Evaluation of STD/HIV/AIDS peer-education and danger: a local perspective. Ciencia Saude Colet. 2008;13(6):1817-24.

5. Santos KB, Murta SG. Influência dos Pares e Educação por Pares na Prevenção à Violência no Namoro. Psicol Cienc Prof. 2016:36(14):787800 .

6. Santos MP et al. Promoting adolescent sexual and reproductive health: peer education. Rev Baiana Enferm. 2017;32(3).

7. Ayres JRCM et al. Adolescence and Aids: evaluation of a preventive education experience among peers. Interface - Comunic Saude Educ. 2003:7(12)113-28

8. BRASIL. Decreto nº. 6.286, de 5 de dezembro de 2007. Institui o Programa Saúde na Escola - PSE, e dá outras providências. Diário Oficial da União, dez. 2007.

9. Santos CMC, Pimenta CAM, Nobre MRC. A estratégia pico para a construção da pergunta de pesquisa e busca de evidências. Rev LatinoAm Enfermagem. 2007:15(3).

10. Assis SG, Gomes R, Pires TO. Adolescência, comportamento sexual e fatores de risco à saúde. Rev Saude Publ. 2014;48(1):43-51.

11. Brasil KT et al. Fatores de risco na adolescência: discutindo dados do DF1. Paidéia. 2006:16(35):377-84.

12. Cerqueira D. Coelho DSC. Democracia racial e homicídios de jovens negros na cidade partida. [acesso em: 16/10/18]. Disponível em: <https://www.researchgate.net/publication/312138855_DEMOCRACIA_RACIAL_E_HOMICIDIOS_DE_JOVENS_NEGROS_NA_CIDADE_ PARTIDA>

13. Rosário AC, Candeias A, Melo M. Violência entre pares na adolescência: Um estudo com estudantes no início e no final do $3^{\circ}$ ciclo do ensino básico. Psicologia. 2017;31(2):57-68. 